\title{
Frequency Adjustment in Ultrasonic Tomography System with a Metal Pipe Conveyor
}

\author{
Javad Abbaszadeh*, Herlina Abdul Rahim, Ruzairi Abdul Rahim and Sahar Sarafi \\ Control \& Instrumentation Engineering Department, Universiti Teknologi Malaysia, \\ Johor, Malaysia
}

(Received November 5, 2012; accepted February 4, 2013)

Key words: process tomography, ultrasonic wave, transmission mode, Lamb wave, ultrasonic tomography, metal pipe

The application of ultrasonic tomography systems for producing cross-sectional images of objects by a noninvasive method plays a dominant role in industry, especially in the oil industry. Since finding the appropriate ultrasonic sensor is the main step in designing an ultrasonic tomography system, determination of the properties of the ultrasonic sensor is very important. In this paper, the manner of ultrasonic wave propagation in various regions and its dependence on frequency are investigated. A finite element software (COMSOL Multiphysics 3.5) is used for visualizing the practical module in a virtual environment to describe the physical interaction inside a metal pipe. Since the transmission mode of an ultrasonic wave is significant in accordance with the data being carried, a frequency should be chosen in which the straight path wave is improved and other propagation modes such as a surface wave on the wall of a metal pipe are attenuated. A frequency of $40 \mathrm{kHz}$ is extracted as the resonance frequency of a desired ultrasonic sensor owing to the simulation results. This claim is proved by the practical testing that is presented in this paper.

\section{Introduction}

A preliminary investigation on determining the component concentration in liquid flow has been carried out in the late 1980s and early 1990s by applying capacitance tomography $(\mathrm{ECT}){ }^{(1)}$ Owing to the application of process tomography in industry, especially for measurements in the oil industry in Europe and the coal industry in the USA, research on process tomography has been carried out. Some of the studies include measurement of water content in oil( ${ }^{(2)}$ and the visualization of component distribution in multicomponent flow pipelines. ${ }^{(3)}$

Principally, an ultrasonic tomography (UT) system consists of two general parts, software and hardware, which are shown in Fig. 1 as a block diagram. The hardware part of UT systems consists of various components, such as a generating pulse,

*Corresponding author: e-mail: JAB@fkegraduate.utm.my 


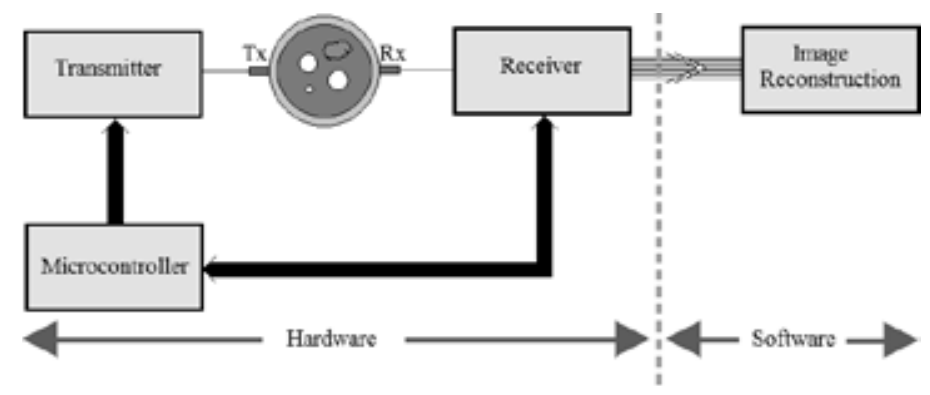

Fig. 1. General block diagram of an ultrasonic tomography system.

amplifying the generated pulse, sending an amplified pulse to sensors that are mounted on the periphery of the pipe to data acquisition and data reception, then reducing the added noise, amplifying the received signal, and finally converting the analog data to digital data. Digitally gathered data in the software part is utilized to construct the crosssectional image of a pipe by applying various algorithms.

The acoustic impedance of layers is the significant point of the design procedure owing to its influence on the manner of ultrasonic wave propagation. The majority of previous research studies to date use the pipes with material such as acrylic, polyvinyl chloride (PVC) or plastic. Owing to the large difference in acoustic impedance among liquid and metal pipes and Lamb wave disturbance, the metal pipe is not investigated as the conveyor in UT. Majority of designers state that it is difficult to use the metal pipes for producing the cross-sectional images in UT process and problems occur owing to reflections within enclosed spaces, such as metal pipes. ${ }^{(4)}$ The main problems of metal pipes are high attenuation of ultrasonic energy and large effect of bulk waves compared with other types of pipe. ${ }^{(5,6)}$

Despite the mentioned problems of a metal pipe as a conveyor of UT systems such as high attenuation property and effect of Lamb wave, we investigated in this study to find the solution. The installation method of ultrasonic sensors and the behavior of ultrasonic wave propagation in different layers are simulated using finite element software. The bulk wave propagation of an ultrasonic wave on the wall of a metal pipe depends on the frequency of the ultrasonic wave. From the simulation results of ultrasonic wave propagation in various frequencies of ultrasonic wave, the optimum frequency for the ultrasonic sensors is estimated.

In this paper, mathematical preliminaries for simulating with COMSOL will be presented in the second part. This is then followed by a demonstration of the simulation results, and the practical structure is described in the succeeding sections. Finally, the conclusion is presented.

\section{Propagation of Ultrasonic Wave}

In this paper, a transmission mode of ultrasonic wave propagation is investigated, and the receiver amplitude and arrival time analysis are emphasized. Arrival time analysis is based on the ultrasonic propagation manner; it takes some time for an ultrasonic 
disturbance to move from one position to another inside the experimental pipe. Another mode of ultrasonic wave (Lamb wave) makes a disturbance in the analysis of the time of flight of a straight path signal. Basically, the longitudinal mode of the ultrasonic wave could penetrate through the pipe from the transmitting sensor to the receiving sensor within a low-acoustic-impedance media such as liquid. For example, the penetration of longitudinal waves from the transmitting sensor $\mathrm{Tx}_{4}$ to the receiving sensor $\mathrm{Rx}_{7}$ is shown in Fig. 2. However, there is another wave generated owing to the complex vibrational effects, which was mentioned as Lamb waves. The Lamb wave is also shown in Fig. 2, which propagates and travels within the pipe boundary.

As consequences of using the transmission-mode method, the amplitude of the receiving wave in the receiver should relate to only the longitudinal mode and not the Lamb mode. The Lamb waves do not provide any information on ultrasonic disturbances caused by the object obstruction inside the pipe because the Lamb wave propagates within the pipe boundary. Finite element simulations with COMSOL software are used for detecting the receiving time of the longitudinal waves and Lamb waves.

Since COMSOL is a mathematical software that can solve wave equations, it is necessary to have an introduction to the mathematics of ultrasound wave, its equations and assumptions. Sound waves in a lossless medium are governed by the following equation for the acoustic pressure, $p$ (with SI unit $\mathrm{Pa}$ ):(7)

$$
\frac{1}{\rho_{0} c_{\mathrm{S}}^{2}} \frac{\partial^{2} p}{\partial t^{2}}+\nabla \cdot\left(-\frac{1}{\rho_{0}}(\nabla p-\boldsymbol{q})\right)=Q .
$$

Here, $\rho_{0}\left(\mathrm{~kg} / \mathrm{m}^{3}\right)$ refers to the density, and $c_{\mathrm{s}}(\mathrm{m} / \mathrm{s})$ denotes the speed of sound. The dipole source $\boldsymbol{q}\left(\mathrm{N} / \mathrm{m}^{3}\right)$ and monopole source $Q\left(1 / \mathrm{s}^{2}\right)$ are both optional. The combination $\rho_{0} c \mathrm{~s}^{2}$ is called the adiabatic bulk modulus, commonly denoted as $K(\mathrm{~Pa}){ }^{(7)}$ An important special case is a time-harmonic wave, for which the pressure varies with time as

$$
p(\mathbf{x}, t)=p(\mathbf{x}) e^{i \omega t},
$$

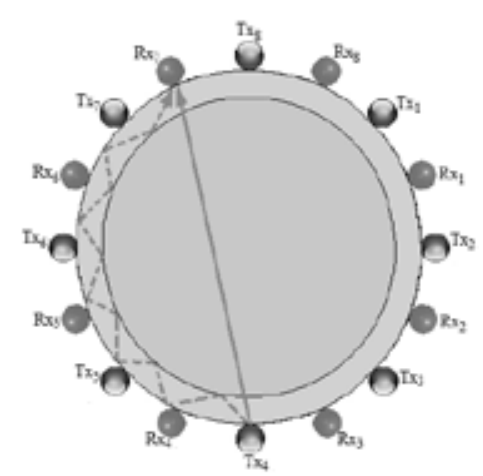

Fig. 2. Two modes of ultrasonic wave propagation in the cross-sectional view of the pipe. 
where $\omega=2 \pi f(\mathrm{rad} / \mathrm{s})$ is the angular frequency, with $f(\mathrm{~Hz})$ denoting the frequency. Assuming the same harmonic time dependence for the source terms, the wave equation for acoustic waves reduces to an inhomogeneous Helmholtz equation: ${ }^{(8)}$

$$
\nabla \cdot\left(-\frac{1}{\rho_{0}}(\nabla p-\boldsymbol{q})\right)-\frac{\omega^{2} p}{\rho_{0} c_{\mathrm{S}}}=Q
$$

In lossy media, it is necessary to introduce an additional first-order term in the time derivative to account for the attenuation of the sound waves: ${ }^{(8)}$

$$
\frac{1}{\rho_{0} c_{\mathrm{S}}} \frac{\partial^{2} p}{\partial t^{2}}-d_{\mathrm{a}} \frac{\partial p}{\partial t}+\nabla \cdot\left(-\frac{1}{\rho_{0}}(\nabla p-\boldsymbol{q})\right)=Q .
$$

For transient analysis, the damping term in eq. (4) is absent from the standard partial differential equation (PDE) formulations in the pressure acoustics application modes. The intensity distribution, $I(r)$, can be obtained from $p(r)$ via the equation: ${ }^{(9)}$

$$
I(r)=\frac{p(r)^{2}}{2 \rho c}
$$

Equation (3) is homogeneous and can be solved by separating the variables. Thus, the final solution can be written as ${ }^{(9)}$

$$
p(r, t)=A e^{i(\omega t-k \cdot \tau)}
$$

The analytical treatment here shows that a sinusoidal function is obtained as the solution to the homogeneous Helmholtz equation. This is in contrast to the results obtained elsewhere, ${ }^{(8)}$ which showed an exponential decay solution with high-intensity regions just below the horn tip. ${ }^{(7)}$ The homogeneous Helmholtz eq. (3) is solved using FEMLAB by the finite element method. It is imperative that, while solving by numerical techniques, the node length $(h)$ should satisfy the following:(9)

$$
\frac{\Delta h}{\lambda} \leq 1
$$

$\kappa \cdot h=$ constant, ${ }^{(10)}$ where $\lambda$ is the wavelength while $\kappa$ is the magnitude of the wave vector and is equivalent to $\omega / c$. This condition is indicative of the accretion of errors in the finite element solution associated with increases in wave number and is termed as the pollution effect. To circumvent this problem, one should use the finest mesh possible, which has been followed in this paper and also elsewhere. ${ }^{(10)}$ 


\section{Simulation Results}

The conveyor of the ultrasonic tomography system is a cylindrical metal pipe with a simple geometry (outer diameter, $20 \mathrm{~cm}$; inner diameter, $18 \mathrm{~cm}$; height, $40 \mathrm{~cm}$ ) with ultrasonic sensors (diameter, $1.5 \mathrm{~cm}$ ), which is implemented on the surface of the metal pipe. The 3D geometry of the pipe with the sensor holder ring and implemented sensors are shown in Figs. 3 and 4, respectively. 2D simulation of the system has been carried out to view precisely the manner of propagation on the boundary of the pipe and inside the pipe. The degree of freedom of meshing in this process is equal to 18220 with the maximum meshing size of 0.012 owing to the ultrasonic wavelength.

According to time harmonic analysis and assuming the resonance frequency of the ultrasonic wave as $20 \mathrm{kHz}$, the simulation result is as shown in Fig. 5. High pressures are denoted by peaks (dark black), whereas low pressures are represented by valleys (light gray).

The propagation of the ultrasonic wave inside the pipe after many reflections with the boundaries is demonstrated in Fig. 5. As can be seen in this figure, Lamb waves can also propagate on the boundary of the pipe considerably. According to this figure, the percentage of Lamb wave to the total amount of ultrasonic wave is a notable
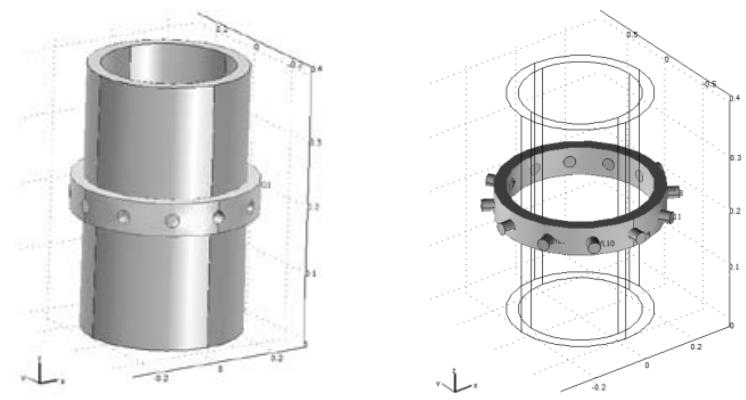

Fig. 3 (left). 3D view of metal pipe.

Fig. 4 (right). 3D implementation of sensors on the surface of metal pipe.

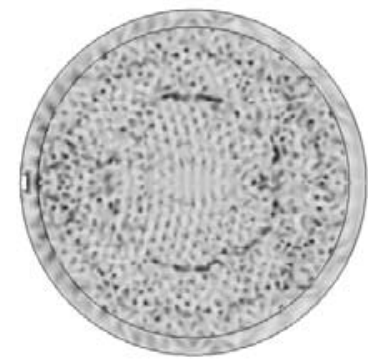

Fig. 5. Ultrasonic wave propagation in $20 \mathrm{kHz}$. 
amount, which can cause a problem in the measurement process. The amplitude of the propagated ultrasonic pressure on the boundary of the metal pipe and inside the pipe is shown as a trend in Figs. 6 and 7, respectively. These trends can be useful for denoting the effect of frequency variation in Lamb wave and longitudinal wave propagation.

The same pressure of ultrasonic wave is applied to the ultrasonic transducer in different frequencies by varying the frequency from 20 to $250 \mathrm{kHz}$. Increasing the frequency from 20 to $250 \mathrm{kHz}$ leads to the decrease in the Lamb wave pressure level as demonstrated in the case of $250 \mathrm{kHz}$ in Fig. 8. As shown in Fig. 8, the length of the Lamb wave propagation is not higher than that in the $20 \mathrm{kHz}$ mode. This phenomenon satisfies the transmission mode measurement in ultrasonic tomography because its amplitude in the receiver part is so weak and it cannot affect the straight path signal. The disadvantage of increasing the frequency is that the longitudinal wave is decayed very fast and it cannot travel a long distance. The mentioned problem is a significant matter because in transmission-mode ultrasonic tomography, the longitudinal wave plays the main role in showing the information of the concentration of the mixed liquid inside the pipe. The trends related to the amplitude of wave propagation in $250 \mathrm{kHz}$, on the wall of the pipe and inside the pipe, are demonstrated on Figs. 9 and 10, respectively. If we compare these trends with previous trends, the effects of frequency variation on the longitudinal wave and Lamb wave are clear.

From the comparison of the ultrasonic wave propagation manner in 20 and 250 $\mathrm{kHz}$, it is concluded that if the frequency of the ultrasonic wave increases, the Lamb wave propagation length decreases. Increasing the frequency reduces the effect of the ultrasonic Lamb wave on the receiver part and this is our desire. On the other hand, increasing the frequency of the ultrasonic wave results in the fast decay of the longitudinal wave.

According to the above-mentioned problem, we should find the suitable frequency that satisfies either the longitudinal-mode or Lamb-mode propagation. From the simulation results in different frequencies, $40 \mathrm{kHz}$ is the most suitable; the trends for the boundary of the metal pipe and inside the pipe are shown in Figs. 11 and 12, respectively.
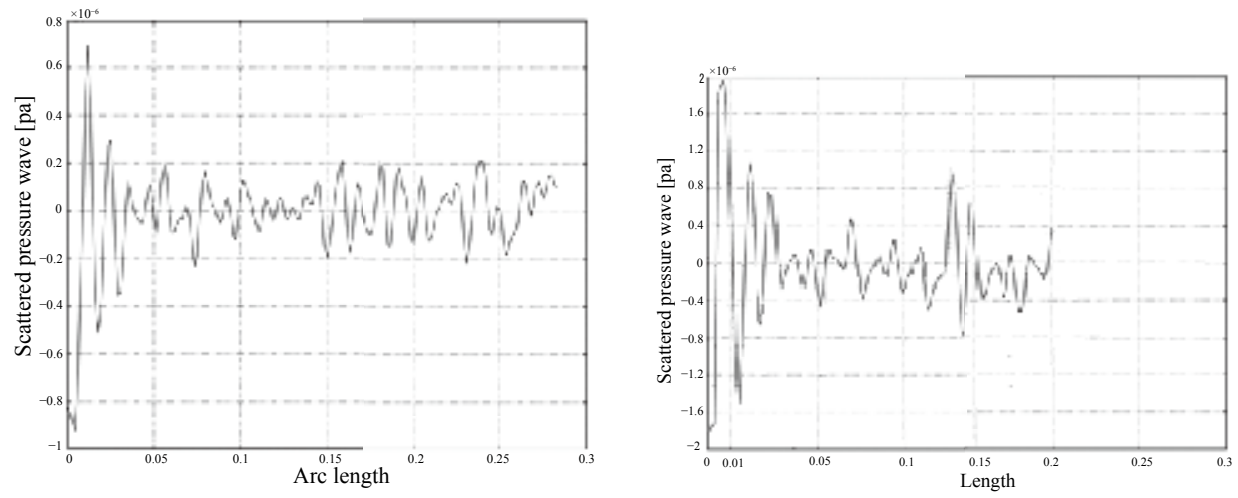

Fig. 6 (left). Distribution of ultrasonic wave on the boundary of the metal pipe in $20 \mathrm{kHz}$. Fig. 7 (right). Distribution of ultrasonic wave inside the metal pipe in $20 \mathrm{kHz}$. 


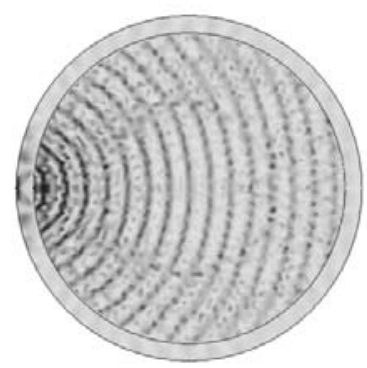

Fig. 8. Ultrasonic wave propagation in $250 \mathrm{kHz}$.
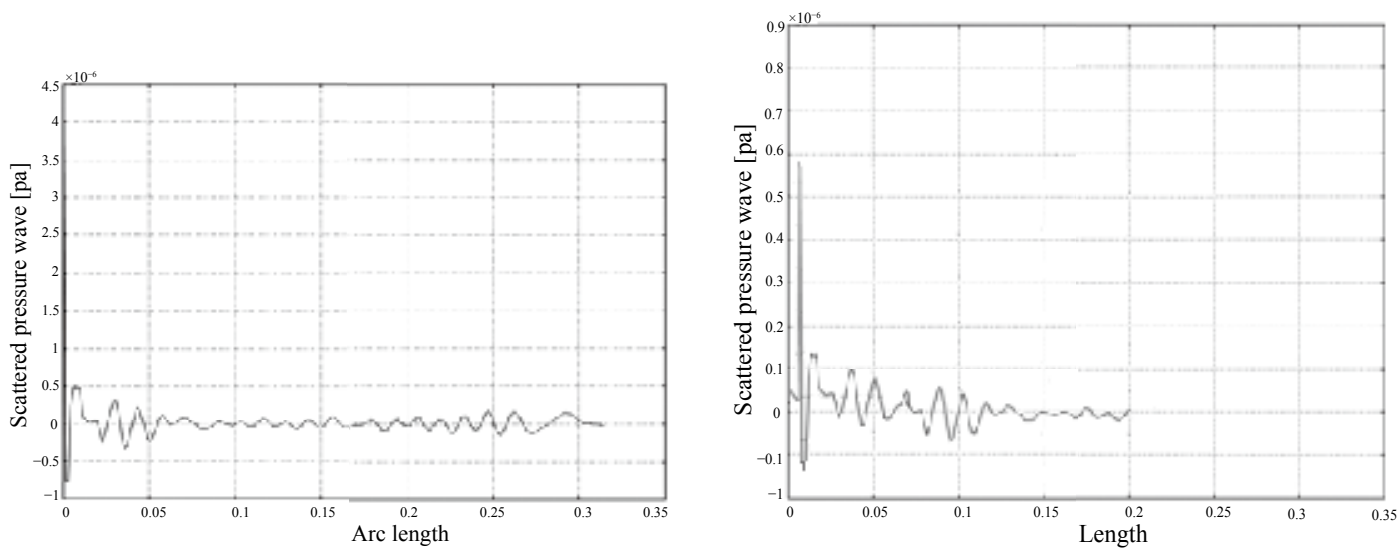

Fig. 9 (left). Distribution of ultrasonic wave on the boundary of the metal pipe in $250 \mathrm{kHz}$.

Fig. 10 (right). Distribution of ultrasonic wave inside the metal pipe in $250 \mathrm{kHz}$.
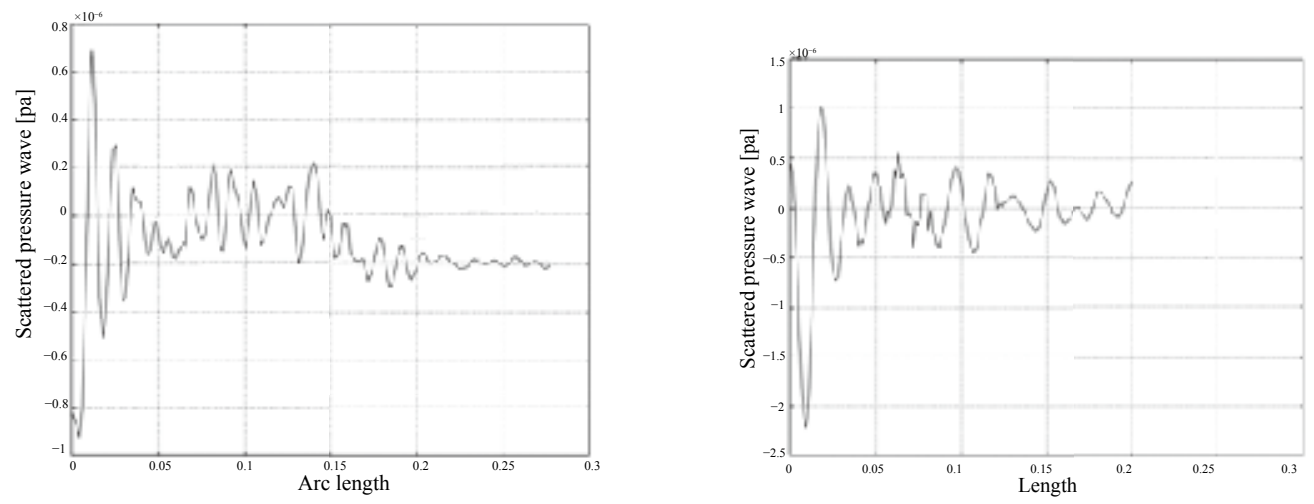

Fig. 11 (left). Distribution of ultrasonic wave on the boundary of the metal pipe in $40 \mathrm{kHz}$. Fig. 12 (right). Distribution of ultrasonic wave inside the metal pipe in $40 \mathrm{kHz}$. 
It is obvious that in $40 \mathrm{kHz}$, both cases are satisfied, and this frequency can be selected as the resonance frequency of the sensor. The sensor applied on the periphery of the metal pipe should have a wide beam angle to cover the wide area of inside the pipe, which can support high-amplitude voltages owing to the attenuation effect of the metal pipe; the resonance frequency of the sensor should be approximately $40 \mathrm{kHz}$.

\section{Practical Structure}

The experimental structure consists of various parts, namely, transmitter circuit for preparing the proper signals with the resonance frequency of ultrasonic sensors, receiver circuit to detect the sent signal from the transmitter and reduce the noise effect and convert the analog signal to digital, and metal pipe with the holding ring of sensors, which is designed using computer-aided design (CAD) software and made with the computer numerical control (CNC) system. The metal pipe with the mounted ultrasonic sensors is demonstrated in Fig. 13. The applied sensors have the resonance frequency close to $40 \mathrm{kHz}$ and cause the simulation criteria to be satisfied. From the results obtained by COMSOL simulation, in $40 \mathrm{kHz}$, the Lamb wave effect decreased markedly and a straight path signal can be detected very clearly. The practical results cover the simulation results and it shows that, in the case of the metal pipe as the conveyor of the ultrasonic tomography system, the $45 \mathrm{kHz}$ frequency is optimum. The whole structure of our designed ultrasonic tomography system is demonstrated in Fig. 14.

\section{Conclusions}

Various noninvasive methods are used to extract the cross-sectional images of the mixed objects in liquid media on the basis of gathered data as the concentration profile of the pipe zone, and then analysis of the extracted image is carried out to determine
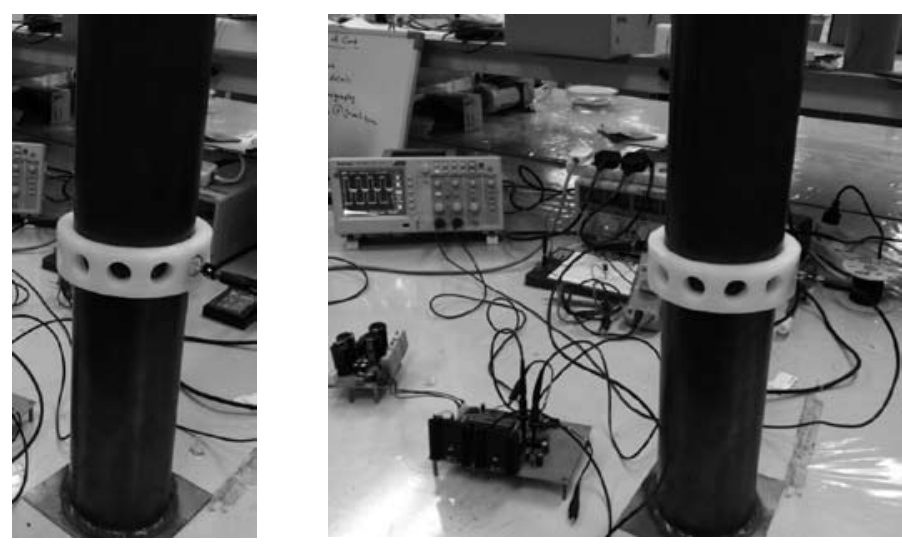

Fig. 13 (left). Metal pipe with holding sensor ring with the mounted sensor.

Fig. 14 (right). Hardware structure of designed ultrasonic tomography system. 
the concentration of liquid/gas/solid mixtures inside the pipe. A popular method that has been used recently is the transmission-mode method. In this method, extracting the concentration profile of inside the pipe is carried out using the straight path signal, which penetrates from the wall of the pipe to inside the pipe.

In this paper, a metal pipe is assumed as the conveyor of the ultrasonic tomography system. Owing to the selection of the transmission mode as the measurement method for the proposed ultrasonic tomography system, the received signal should completely relate to the straight signal. From executed simulations that are carried out using the finite element software (COMSOL), it is found that the plate wave as the Lamb wave disturbs the receiving signal. Increasing the frequency of the ultrasonic wave causes the Lamb wave to have different behaviors. It is concluded that by increasing the frequency, the percentage of the Lamb wave propagation decreases. However, the frequency increase causes the fast decay of the straight-path signal, which is undesirable. Finally, a suitable resonance frequency of the ultrasonic sensor, which can satisfy both modes, is found to be $40 \mathrm{kHz}$.

The resonance frequency of the selected sensor installed on the periphery of the metal pipe for generating the ultrasonic wave is $40 \mathrm{kHz}$ and has been tested practically. The experimental results confirm our assertion that $40 \mathrm{kHz}$ is the optimum frequency of the applied ultrasonic sensor in the case of the metal pipe used as the conveyor of ultrasonic tomography.

\section{Acknowledgements}

This work was partially supported by the Ministry of Higher Education Malaysia and Universiti Teknologi Malaysia under project number R.J130000.7823.4L007.

\section{References}

1 C. R. A. Rahim, N. W. Nyap, M. H. Fazalul Rahiman and C. K. San: Elektrika 9 (2007) 19.

2 H. A. Plaskowski, M. S. Beck, R. Thorn and K. Dyaskowki: Imaging Industrial FlowsApplication of Electrical Process Tomography, (IOP Publishing, Philadelphia, 1995) Chap. 3.

3 M. S. Beck, B. S. Hoyle, M. A. Moris and R. C. Waterfall: Process TomographyImplementation for Industrial Processes (UMIST Publishing, Manchester, 1995) p. 125.

4 M. H. F. Rahiman, R. A. Rahim and M. Tajjudin: IEEE Sens. J. 6 (2006) 1706.

5 B. F. Larson: NDT Education Resource Center Developed by the Collaboration for NDT Education, Center for Nondestructive Evaluation (Iowa State University, Ames, Iowa 50011, USA, 2001).

6 J. Davic and N. Cheeke: Fundamentals and Applications of Ultrasonic Waves (Taylor \& Francis, Inc, Boca Raton, 2002) Chap. 3.

7 J. A. Bargoshadi and E. Najafiaghdam: SPAWDA and Symposium on Frequency Control Technology (IEEE, China, 2009) p. 416.

8 V. Raman: Excerpt from the Proceeding of the COMSOL Users Conference (Proceedings of the COMSOL Users Conference, Bangalore, 2006) p. 1.

9 I. B. Ihlenburg: Comput. Math. Appl. 30 (1995) 9.

10 I. Harari and T. Hughes: Comput. Meth. Appl. Mech. Eng. 87 (1991) 59. 